\title{
From Cornering to Virtual Cornering
}

\author{
Andrea Francesco Martinelli ${ }^{*}$
}

\begin{abstract}
Cornering consists in the assignment of a physical selling space to a producer by a retailer.

Therefore, traditional cornering has the objective of strengthening the value proposition of distribution and industry in the final buyer's mind enhancing the value for the customer by a combination of the efforts inside the physical space.

Traditional cornering is characterised by distinctive specific advantages for the manufacturing companies and for the retailers.

Virtual cornering overcomes the restraints imposed by the physical space of the competition. The ideal environment for virtual cornering is linked to range and offrange products, and to demand bubbles.
\end{abstract}

Keywords: Virtual Cornering; Cornering; Range; Off-Range; Demand Bubbles; Strategic Marketing; Global Markets

\section{Traditional Cornering}

Cornering consists in the assignment of a physical selling space to a producer by a retailer.

Therefore, traditional cornering has the objective of strengthening the value proposition of distribution and industry in the final buyer's mind enhancing the value for the customer by a combination of the efforts inside the physical space.

Traditional cornering is characterised by distinctive specific advantages for the manufacturing companies and for the retailers. In particular, the advantages for the manufacturers concern the following aspects:

- the value of branded products is enhanced - the brand position is strengthened;

- the mix is selected directly by the producer who chooses the products considered to be most appropriate for the corner position and for the available customers target;

- the price is defined by the producer who can choose the most appropriate strategy (Value for money versus Premium price);

- lower distribution costs compared with its own structures;

- assortment strategy (what to display and which rotation to adopt) supervised by the producer;

* Chairman of Metro Cash \& Carry Italia Spa (info@metro-cc.it)

Martinelli Andrea F., From Cornering to Virtual Cornering, Symphonya. Emerging Issues in Management (symphonya.unimib.it), n. 1, 2002, pp. 82-86 
- distribution in market areas or positions which might be difficult to access or difficult to obtain.

Whereas the main advantages for the distribution companies concern the following aspects:

- differentiation in new business areas;

- the distributor acquires new distinctive expertise;

- offer qualification through the 'message' brand of a 'famous' manufacturer;

- lower learning process costs to enter new market segments;

- controlled and contractually defined profitability per square metre.

\section{Cornering Conceptualisation}

To migrate from traditional cornering to virtual cornering first of all requires the manager to be able to conceptualise and to disregard the scenario that he has to face.

However, if we observe merely the physical size of a given event or of a given situation, this becomes a restraint to development and creativity.

However, a sublimation of this tangible aspect occurs when a careful observation of the event stimulates questions from which the formulation of theorems can emerge that may sometimes rise to models that achieve a more universal importance, transcending the tangible form. This conceptual limitation in reality is typical of a managerial culture that focuses on the immediate and tangible solution to operational problems, without preparing a conceptualisation beforehand based on the formulation of strategic thinking.

Virtual cornering has its origin precisely in this conceptual base.

On the other hand, an interpretive effort must be made to identify a number of basic characteristic aspects before formulating a conceptualisation of virtual cornering.

In fact, the consumer is enwrapped in a spectacular way in the case of traditional cornering applied to perfumery and cosmetics present in the large department stores, and is carried into an unreal world of beauty, luxury, pleasantness, compared with the everyday environment, both in terms of material and tangible aspects (such as the lay-out, decor, display, lighting, products), and in terms of intangible sensations (such as a sensation of well-being, a moment of escaping from reality).

Promoters or hostesses themselves are a part of the game in the sense that the way they present themselves and behave make them appear like beings who are absolutely not tied to day-to-day life, to real and tangible existence.

A sense of freedom is experienced, since the purchase advice given is discreet, as well as being professional and educational and the products can be tested in an absolutely free and open atmosphere.

Many senses are involved: touch, sight, smell, and hearing.

There are many novelties, innovative events are numerous therefore it is extremely pleasant, in addition to being relaxing and enjoyable to visit these places.

Once inside, even only just to take a look, the consumer very frequently makes a purchase, perhaps not exactly in the core range of the perfumes assortment or the other range of cosmetics, but of an off-range, inexpensive product, that frequently is also very meaningless and entirely unnecessary and may remain unused for a long time. 
Let's just think how many of us, after entering Sephora on the Champs Élysées, immersed in that special atmosphere after having used perfumes of the most famous and expensive essences have purchased either bath salts or bath marbles filled with emollient oils or gelatin glasses to ease the eyes, which as we have said will remain unused for months and months.

At this point cornering is no longer only a space dedicated to a given commodity, but rises to the level of a system to foster visits and purchases not only in the core range, but also and to a great extent in the off-range. Cornering fosters extemporary purchases (consumption bubbles in a defined and short timeframe), greatly satisfies the need.

All this in a continuously changing and show-like environment, exactly according to the consumer's expectations, because it is already in the consumer's mind as a tangible realisation of something that the consumer had virtualised.

It is essential to observe that one of the major risks which may be experienced when creating a cornering conceptualisation involves the misinterpretation of the other products allocated close to cornering.

In fact, the allocations of these products is frequently the result of too linear and not analysed thought. The result of a too tangible common sense.

That to say, the 'eternal temptresses' of distribution that frequently cause glasses to be positioned next to wine, certain types of cutlery next to the meat, sauces next to pasta with the intention of provoking impulse purchases.

Experience shows that such combinations cause confusion both in the sales area and in the mind of the consumer besides failing to achieve positive economic results, and since they are supplementary to the reference product or category, they run the risk of negatively impacting the respective image and qualification because the range and variety of the assortment is incomplete.

Therefore, cornering is equivalent to a system that before selling products, sells a concept, a sensation which is as relevant as possible to the virtual scenario that the consumer has pre-represented for himself/herself.

Therefore the cornering consists of a complete, broad and exhaustive range of products, related off-range products, a show-like environment, constantly innovative offers on a rotational basis and limited in time, advice that is intended more to strengthen a decision that has already been made rather than focusing on the purchases or to acculturate, the ability to strike the senses, while not affecting the assortment since it enhances both the consumer who can or who wishes to purchase the top range products and the consumer who can purchase lower price products, in fact the top brands will be responsible for also transferring value to other products.

It is advisable to summarise the principle aspects of cornering before outlining the distinctive features of virtual cornering, namely:

- concept;

- consistency to the representation pre-formulated by the final buyer;

- complete range of products;

- consistent off-range mix;

- avoiding being influenced by nearby products;

- discreet advice, no direct selling;

- demand bubbles with a very limited offer timeframe;

- show-like environment. 


\section{Virtual Cornering}

First of all, virtual cornering overcomes the restraints imposed by the physical space of the competition.

It is necessary to define the ideal environment where virtual cornering lives and is enhanced to develop and understand virtual cornering at best by considering the following two basic aspects:

a) range and off-range products;

b) demand bubbles.

\section{a) Range and Off-Range}

'Range' can be defined as everything that is governed by a flow of supply and demand associated with traditional segmentation logic. Therefore, need, monthly breakdown of a given need, segmentation by single category of customers.

The 'off-range' is everything that is not included in the range, that complies with the brand's strategic positioning, even if such positioning must never represent a limit to growth and creativity.

\section{b) Demand Bubble}

The demand bubble represents a flow of resources that feeds the demand curve, moving the curve upwards in the short-term. This avails of two variables: the 'creative offer' variable and the 'timeframe' variable, the demand bubble's principle ally.

At this point we can define virtual cornering as a triggering factor, therefore the factor that originates the demand bubble in an off-range virtual context.

What factors constitute virtual cornering?

The major player is represented by the overall offer, the offer's harmonised structure, associated with its rhythm and its seductive effect, combined with its originality and unique nature.

The offer that uses all the notes on the keyboard of a pianoforte, from the first strong to the last apparently fragile but melodious and equally self-confident. This offer stems from features hidden in the subconscious demand, which awakens and makes them explode. This represents a triumph of gratification which is more sublime than the act of buying that no one wants to give up because the opportunity will not be available later.

The timeframe variable comes into play in this context since there is a beginning and an end, in addition to a rhythmic sequence of 'out of season fruit and early produce'.

How is virtual cornering represented?

Virtual cornering is represented with images (using any form of communication tool) that stimulate and foster the imagination of the general public or virtual segment of persons that share this pseudo-need.

A fundamental aspect which must never be overlooked is represented by the balance and the essential harmony of the products.

In fact, the image must contain a 'merciless' selection of products capable of focusing attention on the offer with a violent impact and at the same time an 'elite' selection of offers that complete the image, enhancing and rendering the image original and unique. 
How is virtual cornering expressed in the stores?

As said previously, virtual cornering overcomes the restraints imposed by the physical space of the competition.

In the case of traditional cornering this aspect is represented by the physical space of store 'packaged' in such a way to begin and end the process, therefore the process originates and is completed at the same time without benefiting from other synergy aspects of the offer. Therefore, all the phases are fulfilled inside the physical space.

Whereas, two phases exist in the case of virtual cornering.

The first phase is represented by the consolidation and belief of something which cannot be renounced by acting on the intangible and psychological factors as we have already described.

This need accompanies the customer hand in hand into the 'container' (store) where the virtual cornering products are contained with other thousands of offers.

With these prerequisites, the store becomes a kind of logistic deposit containing the virtual cornering products, which therefore optimises the space in question and fosters numerous parallel and complementary purchases, all unplanned.

We can conclude by saying that virtual cornering can be a winning factor for retailers since it contains the features of inexhaustability, if wisely guided. 